\title{
LAS ORGANIZACIONES PROFESIONALES Y LAS TECNOLOGÍAS DE LA INFORMACIÓN COMO NUEVOS VALORES DEL PERIODISMO
}

\section{PROFESSIONAL ORGANIZATIONS AND INFORMATION TECHNOLOGY AS NEW VALUES OF JOURNALISM}

Juan Tomás Frutos: Profesor de la Universidad de Murcia. Director de Informativos de TVE en Murcia jtomas.frutos@umd.es

\section{CURRÍCULUM VITAE}

Licenciado y Doctor en Comunicación por la Universidad Complutense de Madrid. Doctor en Pedagogía por la Universidad de Murcia. Está en posesión de seis másteres: Producción en Radio; y Producción en Televisión; Publicidad y Comunicación; Pedagogía; Literatura, y Antropología.

Profesor de la Universidad de Murcia desde hace diez años, donde imparte las asignaturas de Programación Audiovisual, Diseño de Programas en Televisión y Comunicación Interna. Profesor colaborador de varias Universidades extranjeras en Bolivia y México. Ha trabajado como docente en la Universidad Complutense de Madrid y en la Católica de Murcia.

Dirige el grupo de Investigación Universitario “La víctima en los medios”. Trabaja en RTVE desde el año 1989. Actualmente, es Editor de Informativos en el Centro 
Territorial de TVE en Murcia. Con anterioridad ha trabajado como redactor, editor, presentador y Jefe de Informativos en varias unidades y centros de TVE.

Es Decano del Colegio de Periodistas de la Región de Murcia, y Presidente de la Asociación de la Prensa de Murcia desde el año 2007. Es consejero de Redacción de varias revistas digitales.

Ha escrito o participado como coautor en unos 50 libros, entre los que destacan Comunicado sin Claves, Implicaciones, Sensaciones Mediáticas, y La profesión periodística: conceptos y circunstancias. Ha publicado más de mil artículos sobre periodismo y comunicación.

Es escritor, poeta y colaborador intenso en la Asociación de Escritores de la Región de Murcia (AERMU). Actualmente es su Vicepresidente.

\section{RESUMEN}

La pieza básica del desarrollo de toda sociedad que se precie es el Periodismo, un oficio que tiene su origen en la misma idea y en la realidad del ser humano. Cuando éste tiene conciencia de sí mismo y comienza a organizarse en pequeños clanes o familias, ya podemos ver esbozado un primer estadio del Periodismo. En cualquier era podemos detectar a alguien que trata de contar algo a sus congéneres. Es un papel básico, y hasta necesario. La opinión pública precisa de ser alimentada con lo que ocurre y con su interpretación pertinente.

Todo un bagaje instrumental ha rodeado al Periodismo desde que nace de una manera incipiente y poco definida hasta nuestros días. Los avances tecnológicos han sido una consecuencia de las ganas de ir ganando partido a los elementos de la naturaleza, y, en concreto, en el ámbito comunicativo, son utilizados para perseguir 
una mejora en el conocimiento, que es lo que nos distingue, a priori, del resto del medio natural, pues tenemos capacidad de analizar lo abstracto y de vislumbrar lo que ha sido, el porqué, así como su futuro, sus consecuencias y las posibles modificaciones en las conductas y actitudes.

Desde el siglo XVIII, con el fortalecimiento del Periodismo en las urbes, en los burgos, con la nueva clase social, la burguesía, hasta nuestros días, ha habido sucesivos progresos, todos importantes. No obstante, la revolución que supone Internet hoy en día no deja lugar a dudas sobre los avances que estamos registrando. En ellos, el Periodismo encuentra un aliado, si entre todos somos capaces de arbitrar buenas prácticas.

\title{
PALABRAS CLAVE
}

Comunicación - Periodismo - Nuevas Tecnologías - Crisis - Formación

\begin{abstract}
The basic piece of the development of all society considered democratic is the Journalism, a job that has its origin in the same idea and in the reality of the human being. When this has knowledge of himself same and begins to organize in small clans or families, already can be seen a first stadium of the Journalism. In any era we can detect to somebody that treats to explain something to his neighbours. It is a basic paper, and even necessary. The public opinion precises to be fed with what occurs and with its pertinent interpretation.
\end{abstract}

Many elements and instrumentals have surrounded to Journalism since it is born of a way incipient and little defined until our days. The technological advance have been 
a consequence of the desire of winning the party to the elements of the nature, and, in concrete, in the communicative field are used to pursue an improvement in the knowledge, that is what distinguishes us, to priori, of the rest of the natural means, because human being has the capacity to analyse the abstract and all of what has been, the reason why is has happened, as well as its future, its consequences and the possible modifications in the behaviours and attitudes.

From the century XVIII, with the strengthening of the Journalism in the cities, in the villages, with the new social kind, the bourgeoisie, until our days, there has been successive progresses, all important. Nevertheless, the Revolution that supposes Internet nowadays does not leave place to doubts on the advance that we are registering. In that, the Journalism finds an ally, if among all are able to arbitrate good practical.

\section{KEY WORDS}

Communication - Journalism - New Technologies - Crisis - Education

\section{ÍNDICE}

1. La Red ante la crisis: más posibilidades que riesgos

1.1 Sacar provecho de los recursos

2. Internet como instrumento modélico de aprendizaje

2.1 Ir más allá de la sabiduría

3. La profesión periodística, en la encruzijada de la crisis como oportunidad

3.1 Vertebrar y unir al sector periodístico

4. Por un nuevo trayecto periodístico

4.1 Numerosos objetivos 
5. El perfil del periodista desde la óptica de su formación

5.1 Desarrollar todo tipo de habilidades

6. El uso de las tecnologías informativas

7. Fuentes consultadas y Bibliografía

\section{TEXTO}

\section{La Red ante la crisis: más posibilidades que riesgos}

La existencia humana es una cuestión de tener en cuenta el riesgo y la oportunidad en cada etapa histórica, sobre todo cuando hay problemas, desconciertos o desequilibrios. Las TIC'S, como solemos repetir, son las grandes aliadas contemporáneas ante todo tipo de vicisitudes, incluyendo la actual crisis en tantos ámbitos, sobre todo en el económico. La red empieza a influir cada vez más en las compras, sobre todo en las importantes, esto es, a la hora de adquirir una casa, o un coche, o cuando decimos emprender unas vacaciones a buen precio o en el caso de que queramos realizar unos estudios complementarios, y hasta necesarios, para conservar el puesto de trabajo.

Todo se compra y se vende a través de Internet. Superados los recelos iniciales, es constatable el aumento de transacciones que se producen por este sistema, como de ello dan cuenta tanto los Observatorios Regionales de Economía como los informes de las Cámaras de Comercio y de las diversas patronales. Los barómetros particulares de empresas también apuntan en este sentido. 
Conviene recordar que los medios que despiertan más confianza son los periódicos, cuando hablamos de compras y de ventas (y también de otros ámbitos). Además, hemos de subrayar, como se puso de manifiesto en una jornada doblemente convocadas por la Asociación de Editores de Diarios Españoles y el Centro Internacional de Empresas de la Comunicación, cuando hacemos combinación de estas publicaciones con la esfera audiovisual, específicamente con Internet, se incrementan las ventas, la calidad de éstas, la visión y la visibilidad que tienen los posibles consumidores, su valoración, etc. La Red de Redes se ve ya como una oportunidad, como una gran posibilidad de mejora.

Tengamos en cuenta que Internet permite mirar las cosas con más sosiego, a cualquier hora, sin que el espacio ni el tiempo (ni siquiera un vendedor más o menos coercitivo) nos condicionen de manera notable. Las estrategias de relación contractual, de ventas, de compras, de economía en sus diversos géneros o posibilidades, varían, pero está comprobándose que los anuncios en Internet se leen cada vez más, se atienden cada vez más, se comprueban cada vez más, y son cada vez más fiables también. Hasta las entidades más serias, con la banca a la cabeza, confían de manera elevada en las posibilidades de Internet, así como de las publicaciones actualizadas "on line”.

\subsection{Sacar provecho de los recursos}

La crisis se ha convertido en una ocasión para confiar en un instrumento de gestión hasta ahora desconocido u hostil, según se mire. No ha habido más remedio que optimizar costes y tratar de aprovechar lo poco o lo mucho que tenemos, y eso nos ha llevado a costas ignotas, en otro re-descubrimiento de América, en este caso de la Red de Redes, ya no como oportunidad para el espionaje, como en sus inicios, ni para la aventura o el ocio, como ha ocurrido más tarde, sino para saltar a la micro y 
macro economía. La opción, grande ella, se está asumiendo. Sacamos provecho a los recursos materiales, y también a los intelectuales. Corremos con fortaleza hacia una nueva meta, hacia una nueva frontera.

El último Informe Anual sobre la Riqueza en el mundo, avalado y publicado por Merrill Lynch y Capgemini, nos habla de que dos de cada diez ricos han dejado de serlo en los últimos doce meses. La caída de la riqueza, o la mayor concentración de ésta, que las dos cosas están ocurriendo, nos llevan a un nuevo mundo, donde no debemos olvidarnos jamás de los pobres, que siguen creciendo exponencialmente.

Internet se presenta, en ese universo de contradicciones, como una voluntad de constituir una "panacea", siempre y cuando la solidaridad sea bondadosa y plena, algo, por otro lado, difícil. Por donde sí podemos empezar es por la cara de la moneda que nos aproxima a un nuevo planeta en lo financiero, del cual, si sabemos aprovechar las lecciones de los últimos fracasos, todos podemos salir beneficiados (repito, todos). El bajón de actividad nos ha conducido por nuevos derroteros que hemos de convertir, no en derrota, sino en éxito para los que ofrecen y para los que recogen, para los que compran y venden, para un reequilibrio de lo social y, asimismo, de lo económico.

Y no olvidemos que Internet sigue brindando una parte muy ínfima de sus posibilidades para que sigamos creciendo en todas las esferas humanas. Crezcamos. La naturaleza de las cosas, también de la Red, nos impone cambios y mejores perspectivas. La medida ha de ser el obtener beneficios aprovechando todo lo que tenemos al alcance desde una visión ética.

volver al primer apartado volver al principio del artículo volver al principio 


\section{Internet como instrumento modélico de aprendizaje}

El modelo está ahí. De lo que se trata es de usarlo. No cejemos en el intento de vislumbrar las enormes capacidades de este presente-futuro. De vez en cuando hay que mirar el mundo con una cierta garra original. No sé si es el caso que me ocupa, pero de veras que intento expresar una nueva mirada sobre el utillaje de los nuevos medios. Es una visión novedosa, o eso persigue. Lo reconozco, pero seguro que nos sirve para subrayar con abundancia y sin fisuras la fuerza que tienen las Nuevas Tecnologías Informativas. Los avances societarios, en el ámbito de la ciencia, de la mecánica, de la física, de lo material, nos invitan a colocarnos cada vez más en escenarios más cómodos, más fecundos, a la hora de conseguir una formación continua y un reciclaje perpetuo. Las TIC's, en éste y en otros campos, son excepcionales.

Acepto que es una perspectiva un poco particular ésta de la que les hablo a continuación y que se basa en que yo veo a los progresos en las telecomunicaciones, en sus contenidos, en los formatos y soportes, en su adecuación, en los programas, en las panorámicas que nos brindan, como un valor extraordinario para corregir desniveles educativos y/o formativos, pues, a mi juicio, nos facilitan la vida en todo momento y lugar. Uno puede aprender, gracias a las TIC's, cuando quiera, donde quiera, teniendo, claro está, los recursos adecuados, unos recursos que, al menos en el mundo más desarrollado, están a la vuelta de la esquina. De lo que se trata es de conocerlos, de reconocerlos, de ponderarlos, y de sacarles el oportuno partido.

Cada cual se puede preparar una oposición, puede aprender geografía o historia, es capaz de leer la más alta literatura, así como de escuchar música clásica, de viajar por el Imperio Azteca o de acercarse a los enigmas del origen de la vida, por poner unos 
ejemplos, a ritmo de ordenador, de teclas y de deseos, que siempre han de tener el sostén de un conocimiento previo amoldado a lo que queremos seguir escudriñando.

Precisamente, ése es el "quid" de la cuestión. El aprendizaje no se improvisa: es una actitud constante, de puro tesón, de gran voluntad. Hemos de afrontar la vida con el afán de mejorar interior y exteriormente. El equilibrio es fundamental. Por eso, el consejo siempre es no perder las ganas de sorprendernos y de crecer en todas las esferas de nuestras existencias, con la perspectiva de ser las mejores personas del mundo (me refiero al esfuerzo de intentarlo).

\subsection{Ir más allá de la sabiduría}

Tenemos una inmensa suerte en la etapa actual de la sociedad, que, por otro lado, también se caracteriza por un exceso de prisas y de competencias basadas en anhelos financieros y economicistas, suerte, digo, de contar con tantas opciones de conocimiento y para la obtención de informaciones de variada índole. No obstante, seamos realistas: la sabiduría, el disponer de ella, como diría Shakespeare, no es suficiente para ser sabio. Hay que saberla aplicar. Además, a ella no se llega así como así. Debemos fraguar una nueva visión, mucho más amplia en sus horizontes.

Así que, desde la atalaya y la consideración de este artículo, tratamos de hacer hincapié en que las Tecnologías de la Información y la Comunicación son un magnífico taller para reparar los conocimientos adquiridos, para incrementar los ya existentes, para fortalecer memorias e intelectualidades, para encontrarnos y hablar incluso de nuestras esperanzas y posibilidades, que son muchas, las que queramos compartir. 
La primera cuestión que hemos de tener en cuenta es que hemos de potenciar, o reparar, si fuera el supuesto, la ilusión, el entusiasmo por aprender a ser más que a tener. Si disfrutamos la fortuna de conseguirlo, daremos con un cimiento señero para esa felicidad a la que tenemos derecho cuando venimos a este universo de confines tan remotos como cercanos. Hagamos propósito de mirar a todos los inventos como parte de esa Humanidad que precisa reencontrarse consigo misma. Quizá, y eso depende de nosotros, hallemos en tanta tecnología un amigo, o una amiga, insustituible. Adelante. Oteemos las razones que nos asisten, y veremos que no hay motivos para quedarnos parados. En unas décadas, si tenemos en cuenta lo que ha ocurrido en los últimos años, estaremos ante unas posibilidades infinitas de saber. Lo importante es que lo advirtamos ya.

\section{La profesión periodística, en la encruzijada de la crisis como oportunidad}

La crisis económica, y seguramente también de valores, por la que atravesamos en los últimos años ha llevado al Periodismo a una coyuntura llena de incógnitas, pero también de oportunidades, al tiempo que nos planteamos la necesidad de nuevas herramientas de trabajo para mejorar e implementar el panorama actual. Parece lógico que, en cualquier profesión, también en la periodística, nos vayamos dotando de instrumentos emergentes.

Una de las propuestas más importantes realizadas en el marco de la FAPE, la Federación de Asociaciones de Periodistas de España, era y es promover una nueva realidad en nuestras asociaciones profesionales. Nos referimos a los Colegios Oficiales. Con ellos pretendemos tener más visibilidad, más prestigio, más presencia social, al tiempo que nos hemos marcado contribuir a la mejora formativa del sector y a promover una nueva conciencia respecto de nuestra profesión a través de una 
normativa deontológica, que hemos de fomentar y hacer cumplir como base del futuro de nuestro gremio.

Prueba de la buena marcha y de la buena gestión de todo este nuevo proceso es la creación del Colegio Oficial de Periodistas de la Región de Murcia, que comenzó su andadura el pasado 18 de Mayo con su primera Junta de Gobierno. El Colegio ha sido emprendido desde la Asociación de la Prensa de Murcia, que tiene 103 años. Las dos entidades van a existir en conexión, con lo cual se va a unir la impronta y presencia social de un instrumento bien implantado en esta Región a la modernidad de un nuevo organismo, que añadirá estructura y fuerza a un sector necesitado de ambas.

Aunque su vinculación y pertenencia no es obligatoria, aspira a ser una referencia social en los principales asuntos que atañen al sector y que hay que solventar, esto es, la precariedad, los bajos salarios, el intrusismo, la caída de la calidad y de las condiciones de trabajo de los profesionales, las condiciones y circunstancias de las nuevas rutinas laborales que surgen con Internet, etc.

Como quiera que la iniciativa de Murcia posee la virtud de tratar de unir al sector, se observa como un proceso que puede servir al resto de regiones españolas, algunas de las cuales están ya poniendo en marcha procesos similares. Es el caso de Castilla-La Mancha, de Castilla-León, de Madrid, de la Rioja, de Andalucía y de Canarias, que, tras las reuniones mantenidas con sus representantes locales y con sus líderes políticos, han emprendido las negociaciones y las consultas precisas para desembocar en la constitución de unos nuevos colegios, alguno de los cuales podrían ser una realidad a comienzos del año próximo. La Junta Directiva de FAPE ha ido tratando con responsables de las diversas Asociaciones y con los representantes políticos encuentros en este sentido y con el deseo de promover lo antes posible los 
colegios en cada demarcación territorial. Hay un convencimiento profundo de que estas flamantes realidades, que están por venir de manera mayoritaria en este ámbito, supondrán un revulsivo para el sector.

\subsection{Vertebrar y unir al sector periodístico}

Las regiones con varias provincias deben emprender el proceso de "consensuar" con las Asociaciones de la Prensa ya existentes, o bien en el seno de las propias federaciones, la Ley constituyente de sus respectivos colegios, reconocidos como una entidad de vertebración de determinadas profesiones en el artículo 36 de la Constitución española.

El consenso y la unión del sector se tercia como la base de todo este proceso, a la vez que una necesidad, puesto que nos aportan un valor esencial en un momento de especial crisis, que se suma la ya existente en el ámbito de los medios de comunicación desde hace una década.

Tras las reuniones mantenidas desde la Junta Directiva de la FAPE con los Presidentes de las antedichas comunidades autónomas, en proceso de gestación de los colegios, todos han expresado su apuesta por estas nuevas entidades, puesto que consideran que pueden ser, que serán, unos interlocutores válidos a la hora de negociar las situaciones en las que laboran miles de profesionales sometidos a las leyes de un mercado "sin ley".

Como ejemplo de todo este proceso está la Asociación de la Prensa de Madrid, que podría convertirse, al comienzo del próximo año, en el cuarto Colegio de Periodistas de España, junto a los de Cataluña, Galicia y Murcia. Ahora, de lo que se trata es de ajustar mecanismos de conexión con la FAPE, de modo que cumplamos ese aserto de 
que la unión hace la fuerza, en este caso a través de un instrumento que nos puede insuflar más presencia social.

En este sentido, y como demostración de todo el proceso que debemos seguir la FAPE y el Colegio Oficial de Periodistas de Murcia firmarán un acuerdo de vinculación y de colaboración que establezca la cooperación mutua en temas comunes y la representación de sus respectivos intereses en las diferentes esferas de actuación.

La formación será otra de las áreas donde van a tratar de incidir los colegios, y, con este anhelo, la FAPE y las diferentes organizaciones territoriales deben diseñar, y ya están confeccionando, todo un programa de reciclaje profesional y de incorporación a las nuevas tecnologías, al tiempo que, como quiera que hay que recuperar parte de la confianza perdida por el ciudadano, convendría poner en marcha programas de fomento de la lectura de la prensa en la escuela, en sus versiones papel y de Internet, así como planes de acercamiento a la realidad de los medios audiovisuales, en la idea de conseguir una conciencia más pro-activa y crítica a la hora de demandar mejoras en las diversas programaciones y ofertas periodísticas.

Es evidente que hay que emprender mesas sectoriales de trabajo con el fin de contribuir a la mejora de la realidad de los profesionales y de sus correspondientes empresas informativas. Por eso consideramos preciso que demos un impulso a los Colegios, tanto en las regiones donde ya se ha emprendido el proceso, como en aquellas donde hay que apostar por mesas previas de negociación.

Como han demostrado la mayoría de los profesionales consultados en algunas Asociaciones de comunidades como la andaluza o la castellano-manchega, los periodistas demandamos una nueva imagen y una nueva coyuntura para un sector 
que vive despidos y reducciones de personal, y que, con la caída de las audiencias, cada vez más fragmentadas, y de la publicidad y de los impactos de ésta, no apunta hacia un horizonte halagüeño.

Quizá los Colegios no serán la panacea, pero sí son una nueva herramienta de trabajo en un sector que ha pecado de demasiados silencios ante lo que ocurre, $\mathrm{y}$, como se suele decir, el silencio, en éste y en otros casos, no es rentable, nunca es rentable. Los Colegios Oficiales de Periodistas, como dinámica de trabajo en toda España, ya comienzan a ser una realidad. Ahora se trata de dotarlos de contenido, y ahí todos somos necesarios. Todos, todas. Si alguien tiene alguna duda, que trate de hablarla y de comentarla con los demás. El secreto a voces que hemos de hacer realidad tiene que ver con practicar eso en lo que tanto creemos: la comunicación. Miremos con optimismo a un gremio que está en la misma base del crecimiento democrático de las sociedades más avanzadas.

\section{Por un nuevo trayecto periodístico}

Desde el Colegio Oficial de Periodistas de Murcia se anima a la reflexión sobre la coyuntura actual de nuestro sector, amenazado y golpeado por la precariedad, los bajos salarios, la reducción de plantillas que lleva a una merma de la calidad informativa y la desaparición de medios y esperanzas en una profesión que es fundamental en toda Democracia.

Por ello, hemos de poner en marcha una mesa sectorial que nos lleve a ver qué medidas se pueden y se deben emprender. Quizá entre todos podemos contribuir a la hora de arbitrar y de pedir ayudas para el sector, para su reciclaje, para la mejora de su prestigio y de su presencia. 
El Observatorio de la Situación Periodística en la Región nos dará datos para abundar en soluciones que hemos de "consensuar" y de tomar con la perspectiva de que funcionen a medio y largo plazo. El sector debe recuperar la ilusión como instrumento definitivo y definitorio de sus grandes potencialidades, así como en consonancia con la labor de servicio a la sociedad al completo.

No miremos hacia otro lado ante lo que está sucediendo. Todos los días amanecemos con datos escalofriantes de pérdidas de audiencia, de empleos, de publicidad... Muchos amigos y amigas se están quedando sin trabajo o bien su futuro está seriamente amenazado. Los datos nos indican que, al menos, la mitad de los profesionales de la comunicación se plantean dedicarse a otra cosa, y eso no es bueno, ni para ellos, ni para los que han de venir, ni para la propia sociedad. La selección de los profesionales que permanecen en este sector no ha de producirse de esta manera tan dramática.

La frase de "querer es poder" y aquella otra de que "juntos podemos" han de abrirse camino. No es deseable que no reconozcamos el mundo en el que vivimos, con sus circunstancias, con las necesidades de cambios y de mejoras, con las previsiones que hemos de utilizar para determinadas mudanzas, sobre todo a efectos de actitudes. La valentía ha de ser la premisa para que sin prisas, y sin pausas, vayamos hacia otro universo con menos condicionantes en negativo en lo que concierne al Periodismo.

\subsection{Numerosos objetivos}

Entre otros objetivos, hemos de poner en marcha una Cátedra de Comunicación que nos anime a investigar sobre nuestro sector y que, más pronto que tarde, nos sirva de referencia para todo cuanto hay que hacer. Decían los griegos que en el punto intermedio está la virtud. Por eso, parece lógico que intentemos corregir los extremos 
carenciales en los que se hallan muchos compañeros. Se habla de la destrucción de un 30 por ciento de los empleos, de salarios que no llegan, como media, a los 600 euros al mes, de jornadas extensas sin compensaciones, como ocurre en otros oficios, de demasiados fines de semana laborando al mes, de falta de tiempo (con todo este panorama parece normal) para formarnos....

Es momento, pues, de coger otro tren, de dirigirnos a otra estación en la que compartir ese diálogo necesario, seguido de actuaciones y de propósitos de enmienda, para salir de ese túnel carente de la suficiente dignidad laboral. Creo que en ello estamos de acuerdo todos, y por eso todos nos hemos de aproximar a ese espacio donde nos sintamos lo más cómodos que sea posible para afrontar una nueva etapa en la que, por supuesto, no sobra nadie. Todos somos esenciales para arreglar las disfunciones de un trabajo que, pese a todo, sigue siendo el más hermoso del mundo.

\section{El perfil del periodista desde la óptica de su formación}

En esta etapa nuestra en la que el saber apenas ocupa un lugar físico, pues todos los formatos reales, virtuales o de registro se aminoran más y más, sin olvidar que todo lo conocido está al alcance de un teclado, es obvio que hemos "relativizado" más de la cuenta el valor de la formación. Parece que ésta puede aparecer con un chasquido de los dedos, o con solo desearla. No es así, no es tan sencillo.

La formación, el aprendizaje, es algo que llega poco a poco, con una cierta lentitud, que hace que la cultura, la verdadera cultura, se vaya posando en nuestra manera de ser y de comportarnos. Además, ese poso se consigue con el paso de los años, con la experiencia, que también empieza a ser una cierta cenicienta en el ámbito en el que nos hallamos. 
Siempre se ha dicho que la primera docencia es útil a lo largo de la historia de cada persona. Lo es porque nos impregna de valores, de tesón, de armonía y deseo por aprender, de voluntad, de ritmo, de itinerario para el estudio, etc. Por eso conviene insistir tanto en ella. Luego, cuando ya somos mayores, es la Universidad, si llegamos a este estadio, la que nos puede proporcionar un gran impulso, un segundo gran impulso, en la formación integral que nos ha de caracterizar en el ámbito profesional, y también, en esencia, en lo personal.

Así es. La formación universitaria es fundamental en una sociedad. Lo es siempre, y en todo lugar, porque ayuda a sostener los valores democráticos. Lo es aún más, si cabe, en el caso de los estudiantes de Comunicación, pues contribuye a un buen desempeño de la profesión de periodista y de los diversos quehaceres en las diferentes ramas o especificidades de un campo tan amplio como heterogéneo y atractivo. Una buena formación sirve de nexo al estudiante entre sus ilusiones y deseos y el mercado laboral que para él, en esa etapa de su vida, está a la vuelta de la esquina, y para el cual quiere y debe prepararse.

Por ello, esta carrera universitaria ha de tener muy bien equilibrados los aspectos teóricos y los prácticos, y, así, se debe ofrecer un aprendizaje lo más cercano a lo que un poco más tarde se van a encontrar los alumnos a la hora de afrontar un trabajo en este campo.

La capacitación profesional, junto a un sustento teórico interesante, es fundamental para que la incardinación oportuna del estudiante sea del modo más adecuado. Es preciso para ello que haya un mayor acercamiento entre el mundo laboral y el académico. La presencia de profesionales de empresas en la Universidad y la exposición de motivos y de aspectos formativos por parte del profesorado en las mismas empresas son dos ejes cruciales para que haya un entendimiento entre dos 
realidades que están llamadas a un entendimiento recíproco. Durante años ha habido, más en algunas carreras que otras, un distanciamiento entre las necesidades y los planteamientos, entre lo que se sabía y lo que se exigía para una conveniente colocación y ubicación en el mercado laboral. No debe seguir ocurriendo.

Ahora, en pleno siglo XXI, de lo que se trata es de romper las malas inercias, que, cada vez, afortunadamente, son menores, así como de afrontar la realidad de las necesidades formativas desde algo más que el voluntarismo mal entendido o poco prometedor. Precisamos conocer mejor los sectores donde se incardinan los estudiantes, al tiempo que hemos de saber los aspectos más fuertes y los más débiles del aprendizaje que les damos. No finjamos preocupaciones que no ejercemos, y pongámonos manos a la obra.

Debemos contactar con las empresas, con sus empleados, con sus responsables, y hemos de exigir de todos ellos informes que den cuenta de las ventajas y desventajas, de los vacíos y de las fortalezas que se detectan en las formaciones de los estudiantes que les enviamos a esas mismas organizaciones tanto a nivel de becarios o como en lo que se refiere a las prácticas durante su formación universitaria, teniendo presente que, más tarde, han de conseguir una relación laboral mucho más estable y mejor remunerada.

Tendríamos que hacer, desde las Universidades, seguimientos de las habilidades y capacidades que hemos desarrollado en nuestros alumnos. Habría que analizar los progresos que se desarrollan y los motivos de los triunfos o fracasos que se puedan suceder. La Comunicación, solemos repetir, es todo, y no podemos dejar ese todo en un momento temporal determinado. Debe haber un seguimiento. 


\subsection{Desarrollar todo tipo de habilidades}

Con el mayor conocimiento de la realidad en todos los ámbitos y/o esferas que demandamos podemos conseguir ver con qué medios reales se trabaja, al tiempo que podemos aprender sobre los sistemas o modelos de laboriosidad que se utilizan de manera preferente. La Universidad, en determinadas profesionales como la de informador, periodista, comunicador, relaciones públicas, publicista, etc., debe preparar alumnos que tengan unas grandes dosis de teoría, pero, al mismo tiempo, sin que parezca una capacitación de taller neta la que procuramos, hemos de defender un aprendizaje que invite y permita que los alumnos no vean las diversas rutinas de trabajo como algo que solo conocen por libros, si es el caso. El saber, creemos, ha de ser universal, integrador, forjado desde las raíces mismas del deseo solidario de compartir la experiencia, la ciencia, la técnica, todo lo que nos enriquece.

La Universidad debe tener la habilidad de desarrollar unas materias y unas líneas de aprendizaje lo más apegadas a las necesidades reales, que, en primer término, hay que averiguar, así como todos debemos adaptarnos a ellas. Aún distinguiendo entre la capacidad profesional y la investigadora, que han de ser complementarias, con el propósito de hacer rentables las dos en la medida de lo posible, es claro que hemos de adecuar y de amoldar la docencia a lo que existe en el mercado, a sus demandas, a su realidad cotidiana. No olvidemos tampoco que el perfil curricular no se queda en la enumeración de las asignaturas, sino en las proyecciones reales de sus nomenclaturas.

Las metodologías han de basarse en aspectos teóricos, en lecturas frecuentes y variadas, en lo que piensan y defienden profesores de toda índole y calado, pero, asimismo, han de sustentarse en una cercanía a las necesidades de los mundos económico y laboral. Hemos de hacer visitas, así como debemos procurar que las 
prácticas docentes tengan como finalidad publicaciones y emisiones de los mejores trabajos.

Como quiera que los medios y los recursos, amén del profesorado, son escasos (siempre lo son), conviene que establezcamos planes de implantación, de búsqueda de bases para el desarrollo profesional e investigador, así como estrategias para priorizar y para establecer los objetivos claves. Aún no buscando resultados perfectos hemos de perseguir la excelencia y el fomento de algo tan intangible como necesario: la ilusión por el desempeño ético de una profesión que ha de establecer la virtud en el equilibrio entre la técnica y la humanidad. Nos debemos a ello, y, en ningún caso, hemos de renunciar a esa convergencia.

El esfuerzo en las próximas décadas, y para ello debemos empezar a esforzarnos ya, debe ser el dirigirnos al unísono atemperando los ritmos del aprendizaje de modo que todos podamos "empatizar" desde la sana moderación y con un balance oportuno entre lo teórico y lo práctico. Todo es bueno cuando el propósito, la intención y los resultados lo son. Busquemos sin prisa, y sin pausa, los efectos más queridos de la docencia y del aprendizaje, fundamentalmente en una profesión tan de servicio público como es la de comunicador, la de periodista. Todos nos sentiremos reconfortados por ello.

No olvidemos que el derecho que no ejercemos no se tiene. Confiemos en que la experiencia, que es "la madre de la ciencia", según el saber popular, permita que vayamos viendo ese bosque de buenas intenciones y de mejores perspectivas donde nos aguarda la felicidad del aprendizaje desde el anhelo de compartir lo que tenemos, sobre todo si es intelectual. Miremos hacia delante. 


\section{El uso de las tecnologías informativas}

La información es, o debe ser, la misma, pero su puesta en escena, su desarrollo, es otro. Lo es por los grandes avances. Internet es la gran revolución. Lo es, lo ha sido, y lo será, para todos los sectores de la sociedad, sobre todo para aquellos que tienen como base la transmisión de datos y de información. Por eso conviene ver el impacto y la incidencia que van teniendo en ciertas labores, como la periodística.

Los resultados de algunos estudios, como el que mencionamos en esta oportunidad, son muy positivos.

Un estudio realizado entre 84 profesionales de la comunicación de la Región pone de manifiesto el uso extendido de las nuevas tecnologías informativas entre estos trabajadores, que ven en las TIC's, como no podía ser de otro modo, un instrumento de ayuda en sus respectivos trabajos, así como un apoyo a la hora de contrastar noticias y de buscar fuentes para sus noticias y reportajes. Todo avanza a un ritmo galopante, y también lo hace la utilización de Internet y de los ordenadores, con todos sus soportes, formatos y posibilidades. Los periodistas lo saben, y por ello hace ya más de una década que se han venido implantando estos avances sin pausa, buscando optimizar en todo momento los recursos. El estudio al que nos referimos fue realizado en el marco de un análisis de la Facultad de Educación de la Universidad de Murcia, dirigido por el catedrático Pedro Guerrero sobre la situación de los periodistas.

Por hacer una reseñable comparativa de datos, señalemos que un estudio de la Asociación de la Prensa de Murcia del año 2002 da cuenta de la necesidad que entonces, como ahora, se planteaban los periodistas en el aspecto formativo y en relación concretamente a las nuevas tecnologías. Veían las TIC's como una oportunidad y como una necesidad, dos caras de la misma moneda. Sin riesgo, 
decían los antiguos, no hay ocasión. Y la ha habido: en unos años se han incardinado estas tecnologías en el sector periodístico, como en otros, y tanto en cuanto a su uso como fuente de información y transmisora de datos, como en cuanto a los soportes para la difusión de noticias, reportajes y todo tipo de géneros periodísticos.

Llegado el año 2008 conocimos que el 81.2 por ciento de los periodistas de la Región indican, según la mencionada encuesta, que hacen uso de Internet, y añaden, igualmente, que la Red de Redes ha mejorado su capacidad y su nivel profesional. Está claro que, como complemento, Internet se ha vuelto básica. Se gana tiempo, se consultan otros pareceres, otros objetivos, datos e intereses, y, en este sentido, ya no hay una unidad de criterios, sino muchos criterios. Ahora el profesional debe saber discernir lo importante de lo que no lo es, lo relevante de lo anecdótico, lo global de lo parcial, para ayudar a la sociedad a que comprenda las claves que nos conciernen a todos como individuos y como colectivo.

También se consideran los profesionales formados en materia de nuevas tecnologías (más de un 70 por ciento), si bien todos, en el apartado de observaciones de la muestra aludida, expresan la conveniencia de hacer más cursos, de más calidad, de más duración, fundamentalmente en el ámbito de las nuevas tecnologías y de los diversos programas. Está claro que es éste un proceso imparable donde todos convenimos que la formación ha de ser permanente y durante toda la vida. No obstante, hay un 42 por ciento de empresas que no suelen hacer cursos de reciclaje de ningún tipo, y en ese campo es preciso que todos trabajemos, especialmente las organizaciones profesionales. Debemos convencernos de esta necesidad, del valor añadido de este reciclaje.

La convergencia a la que nos lleva Internet de todos los medios existentes hasta ahora, esto es, la prensa, la radio y la televisión, supondrá ciertas adaptaciones de los 
géneros y espacios que hasta ahora hemos conocido. Únicamente así ganaremos con las tecnologías y con sus progresos, pues podremos optimizar y rentabilizar los recursos de los que disponemos. Lo que queremos decir es que no ha de haber una mera traslación de formatos, sino una mejora de éstos con "el nuevo gran soporte". La experiencia de los últimos años ha de servir en esta esfera. También dice la reseñada encuesta que hay que trabajar para que los periodistas sepan rentabilizar los abundantes instrumentos de labor con los que contamos. El conocimiento precisa más conocimiento.

La mejora en las fuentes, como se ha dicho, y también en la consulta de eventos casi en tiempo real, sin olvidar las enormes posibilidades formativas y culturales que posee Internet, entre otras cosas por su permanencia en el tiempo, son los grandes aliados con los que hemos de caminar en el futuro inmediato de la profesión periodística. Será, como ocurre en otros ámbitos, el uso que hagamos de este gran medio de comunicación el que decida si prevalecen o no sus bondades. Por lo tanto, está en nuestras manos lo que queremos que sea, lo que deseamos que nos aporte. De nuevo, el ser humano debe hacer un buen uso de las máquinas y de sus engranajes. Aprovechemos otros errores históricos. En el fondo, estamos hablando, como en otros escenarios, de que el ser humano es la medida de todas las cosas. Si no hacemos exclusiones, este aserto funciona.

\section{Fuentes consultadas y Bibliografía}

Fuentes Consultadas:

-Campus Digital, Revista Electrónica de la Universidad de Murcia. 
-Portal Infoamérica, auspiciado por la Cátedra de Comunicación de la UNESCO y por la Universidad de Málaga.

-SICARM 2.009, Conclusiones de la última edición de este encuentro de profesionales, alumnos y medios de comunicación en torno al Periodismo Digital.

-Ventana Digital de la Región de Murcia, publicación virtual editada por la Fundación Integra.

Bibliografía:

-ISLAS, Octavio; BENASSINI, Claudia: Internet. Columna Vertebral de la Sociedad de la Información. Monterrey, 2005. ITESM.

-MARCOS RECIO, J.C.: La documentación electrónica en los medios de comunicación. Madrid, 1999. Fragua.

-SALAVERRÍA, Ramón: Redacción Periodística en Internet. Pamplona, 2005. EUNSA.

-WARD, Mike: Journalism on line. Oxford (Reino Unido), 2002. Focal Press. 